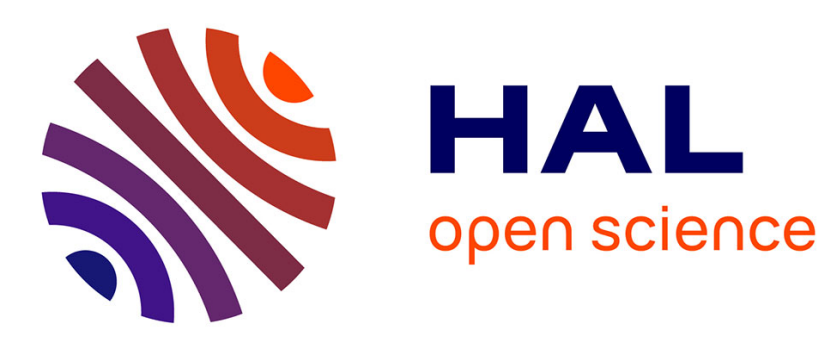

\title{
Diderot et l'approche déterministe de l'esprit : un autre déterminisme?
}

\author{
Charles T. Wolfe
}

\section{To cite this version:}

Charles T. Wolfe. Diderot et l'approche déterministe de l'esprit: un autre déterminisme?. DixHuitième Siècle, 2014, 46, pp.132-146. 10.3917/dhs.046.0501 . hal-01232652

\section{HAL Id: hal-01232652 \\ https://hal.science/hal-01232652}

Submitted on 4 Dec 2015

HAL is a multi-disciplinary open access archive for the deposit and dissemination of scientific research documents, whether they are published or not. The documents may come from teaching and research institutions in France or abroad, or from public or private research centers.
L'archive ouverte pluridisciplinaire HAL, est destinée au dépôt et à la diffusion de documents scientifiques de niveau recherche, publiés ou non, émanant des établissements d'enseignement et de recherche français ou étrangers, des laboratoires publics ou privés. 


\section{Diderot et l'approche déterministe de l'esprit : un autre déterminisme ?}

à paraître dans Dix-huitième siècle (accepté en octobre 2013)

Commençons par un constat qui dépasse les limites de l'âge classique ou la période des Lumières qui nous concerne ici : il existe au moins un penseur de l'époque qui affirme un déterminisme entier et radical - un univers causalement clos sans acte libre «non causé » - et en même temps affirme l'existence d'un hasard irréductible à des schémas de prévision. Ce penseur, c'est Diderot, qui dans des textes célèbres notamment dans la Lettre sur les aveugles, et le Rêve de D'Alembert articule une sorte de cosmogonie transformiste d'inspiration atomiste et particulièrement lucrétienne; le Rêve de D'Alembert devait initialement s'intituler Rêve de Démocrite ${ }^{1}$. Plus précisément, Diderot élabore une forme hérétique du déterminisme à partir d'une réflexion typiquement libertine inspirée par Lucrèce, Hobbes et Spinoza (parmi d'autres), chez Fontenelle dans son Traité de l'âme (1700, mais en circulation dans le volume intitulé Nouvelles libertés de penser à partir de 1743) et dans la Lettre de Thrasybule à Leucippe, habituellement attribuée à Nicolas Fréret.

Il me faut tout de suite justifier le choix terminologique du terme « déterminisme ». Contrairement à un lieu commun maintes fois répété, « déterminisme» ou plutôt «Determinismus » existe bel et bien au $18^{\mathrm{e}}$ siècle $^{2}$. Le mot existe, même s'il est vrai qu'on opposait plutôt la liberté à la nécessité, comme dans l'échange entre Hobbes et l'évêque Bramhall, Of Liberty and Necessity (mais il date du milieu du $17^{\mathrm{e}}$ siècle). Dans son livre de 1793 Die Religion innerhalb der Grenzen der blossen Vernunft, Kant critique l'expression «Determinismus » et préfère celle de «Praedeterminismus »; Hume et Charles Bonnet parleront de la «détermination » de l'âme ou d'un objet par les lois du mouvement, à la suite de Leibniz. On trouve le terme même en titre d'un ouvrage de 1789, Determinismus und moralische Freiheit de Christian Wilhelm Snell. Et, comme on le

\footnotetext{
${ }^{1}$ Ainsi qu'il l'écrit à Sophie Volland, le 2 et 11 septembre 1769. Pour être précis, Diderot pensait nommer les trois interlocuteurs Démocrite, Hippocrate et Leucippe, sous l'influence de la Lettre de Thrasybule de Leucippe (Naigeon, Mémoires historiques \& philosophiques sur la vie \& les ouvrages de Diderot, Paris, Brière, 1821 ; reprint, Genève, Slatkine, 1970, p. 213).

2 On retrouve partout cette affirmation; par exemple: G. Stenger, Nature et liberté dans la pensée de Diderot après l'Encyclopédie, Oxford, Voltaire Foundation, 1994, p. 188 ; J. Harris, Of Liberty and Necessity. The Free Will Debate in Eighteenth-Century British Philosophy, Oxford, Clarendon Press, 2005, p. 7.
} 
verra plus loin, le célèbre passage de Laplace qui donne la définition canonique du déterminisme remonte aux années $1770^{3}$.

Le siècle employait évidemment le terme de «fatalisme». Mais, de Fontenelle à Collins, de L'Âme matérielle à Diderot, l'ensemble de textes ou «tradition souterraine » étudiée ici ne s'intéresse pas à la thèse (fataliste) selon laquelle nos actions serait inutiles ou superflues, et reconnait que les événements à venir sont, au moins en partie, produits par nos actions (une position pleinement déterministe) ${ }^{4}$. Le fatalisme est une forme laïcisée de l'idée ancienne selon laquelle, pour citer Madame de Staël, « la fatalité venait de la volonté des Dieux », alors que «chez les modernes, on l'attribue au cours des choses »: dans le fatalisme moderne, «si les circonstances nous créent ce que nous sommes, nous ne pouvons pas nous opposer à leur ascendant ; si les objets extérieurs sont la cause de tout ce qui se passe dans notre âme, quelle pensée indépendante nous affranchirait de leur influence ? $\gg^{5}$. Au contraire, la forme du déterminisme que nous cherchons à reconstruire ici s'attache au rapport entre notre corps, notre vie mentale, et nos actions.

Cet «autre» déterminisme s'oppose à sa forme mieux connue, qui est essentiellement laplacienne. Celle-ci est de nature physicaliste, axée sur la prévisibilité des événements, réduits à des rapports de localisation entre des particules et des forces. En effet, l'intelligence suprême de Laplace, comme le démon de Maxwell qui lui succèdera, possède une connaissance totale de l'univers si elle saisit « toutes les forces dont la nature est animée et la situation respective des êtres qui la composent ${ }^{6}$. La thèse fondamentale du déterminisme laplacien est alors que tout événement a une cause, et plus encore, que l'état actuel de l'univers est la cause de son état à venir. Dans un univers défini par la mécanique newtonienne, tout est explicable en termes de corps, de forces, et de masses, qui sont eux-mêmes réductibles à un système de points matériels caractérisés par des coordonnées de position $q$ et des quantités de mouvement $p$; il suffit alors de connaître toutes les causes naturelles et la position de chaque corps, pour pouvoir calculer l'intégralité des événements futurs.

\footnotetext{
${ }^{3}$ R. Kuhlen, C. Seidel et N. Tsouyopoulos, « Determinismus / Indeterminismus », dans J. Ritter et al. dir., Historisches Wörterbuch der Philosophie, Bâle/Stuttgart, Schwabe, 1972, vol. 2, p. 151.

${ }^{4}$ Nous empruntons cette distinction utile entre fatalisme et déterminisme à M. Dorato, « Determinism, Chance and Freedom », in Between Chance and Choice, dir. H. Atmanspacher \& R. Bishop, Exeter, Imprint Academic, 2002.

5 G. de Staël, De L'Allemagne [1810-1813], III ${ }^{\mathrm{e}}$ Partie, ch. 1 («La philosophie »), Euvres complètes, Bruxelles, Wahlen, 1820, vol. IX, p. 135-136.

${ }^{6}$ P.S. de Laplace, Essai philosophique sur les probabilités [1814], Paris, Gonthiers-Villars, 1921, p. 3.
} 
Mais l'affirmation selon laquelle il a existé un «autre déterminisme », qui ferait une place au hasard et à des événements spécifiquement mentaux, devrait faire sursauter, non plus les gardiens de l'orthodoxie terminologique, mais tout philosophe digne de ce nom. Comment employer le terme « déterminisme » pour une doctrine qui ferait une place au hasard, ou à des événements mentaux, puisque, comme le formule avec bon sens Cournot, «Ce qu'il y a de fondamental [...] dans la notion de hasard c'est l'idée de l'indépendance, ou de la non-solidarité entre diverses séries de causes $»^{7}$ ?

Or, il existe bel et bien une différence entre un déterminisme axé sur la calculabilité des états de l'univers et donc leur prévisibilité, et un déterminisme qui laisse une place, non pas à la liberté mais à l'aléatoire. Le premier, un déterminisme physicaliste axé sur le calcul et la prévision, n'est pas non plus un pur produit de la physique du $19^{\mathrm{e}}$ siècle, comme nous l'avons indiqué précédemment. Certes, la $1^{\text {ère }}$ édition de $1^{\prime} E s s a i$ philosophique sur les probabilités de Laplace date de 1814 (la dernière revue est la $5^{\mathrm{e}}$ édition, de 1825); mais non seulement le texte reprend un cours donné à l'École Normale en 1795 ; de plus, déjà en 1772 Laplace avait lu un mémoire devant l'Académie des sciences sur le calcul intégral et le «système du monde». Des expressions et des idées semblables apparaissent dans l'article « FORTUIT » de D’Alembert, mentor de Laplace, qui raisonne sur le caractère systémique et interdépendant du système du monde, et parle du calcul d' «une intelligence » suprême et générale (Enc. VII, p. 205a ; publié en 1756). Enfin, des textes très proches qui parlent par exemple de calculer l'action d'un « tourbillon de poussière » à partir de ses particules se trouvent dans la Lettre sur le système du monde de Condorcet (1768), le Système de la nature de d'Holbach (1770) et même ... un fragment de Diderot. Voici d'Holbach :

Dans un tourbillon de poussière qu'élève un vent impétueux, quelque confus qu'il paraisse à nos yeux, [...] il n'y a pas une seule molécule de poussière qui soit placée au hasard, qui n'ait sa cause suffisante pour occuper le lieu où elle se trouve, et qui n'agisse rigoureusement de la manière dont elle doit agir. Un géomètre qui connaîtrait exactement les différentes forces qui agissent dans ces deux cas, et les propriétés des molécules qui sont mues, démontrerait que, d'après les causes données, chaque molécule agit précisément comme elle doit agir, et ne peut agir autrement qu'elle ne fait ${ }^{8}$.

\footnotetext{
7 A.-A. Cournot, Essai sur les fondements de nos connaissances [1851], Paris, Vrin, 1975, §32.

8 D'Holbach, Système de la nature [1770, $2^{\mathrm{e}}$ édition 1781], éd. J. Boulad-Ayoub, Paris, Fayard-Corpus, 1990, I, ch. iv, p. 19. On trouve des passages analogues chez Diderot, y compris un fragment du fonds Vandeul cité par G. Stenger, qui propose lui aussi, un Gedankenexperiment bien 'proto-laplacien' : «Si la somme peut-être infinie des attributs, de la multitude peut-être infinie des molécules de la nature nous était parfaitement connue, il m'est évident que nous verrions tous les phénomènes s'exécuter par des lois rigoureusement géométriques... » (Diderot, Inventaire du fonds Vandeul et inédits de Diderot, éd. H. Dieckmann, Genève, Droz, 1951, p. 255, cit. Stenger, Nature et liberté dans la pensée de Diderot, p. 208).
} 
Et le physicalisme de Hobbes, selon lequel les idées ne sont que des petits mouvements dans le cerveau, partage avec le déterminisme laplacien la non-reconnaissance d'une causalité spécifiquement mentale.

Quant à l' «autre déterminisme », « hasardeux » (comme on dit «matérialisme aléatoire »), il apparait chez Diderot dans le sillage de la tradition libertine et sa relecture de motifs atomistes, mais aussi, nous espérons le montrer ici, dans un processus de complexification du schème causal qui reconnait la spécificité de l'action et de l'activité mentale. Ce processus commence avec Locke et son chapitre sur le Pouvoir dans l'Essai concernant l'entendement humain ${ }^{9}$, se poursuit chez son disciple radical, le déiste Anthony Collins, dans son Philosophical Inquiry Concerning Human Liberty de 1717 (traduit deux fois en français au cours du $18^{\mathrm{e}}$ siècle, avec un retentissement dont la réaction de Voltaire nous donne une idée : «cette question sur la liberté de l'homme m'intéressa vivement; je lus les scolastiques, je fus comme eux dans les ténèbres; je lus Locke et j'aperçus des traits de lumière; je lus le traité de Collins, qui me parut Locke perfectionné; et je n'ai jamais rien lu depuis qui m'ait donné un nouveau degré de connaissance $\left.{ }^{10}\right)$. Ce déterminisme particulier est développé ensuite au sein de traditions de plus en plus distinctes par Diderot, qui «biologise » le déterminisme, et par Hume, Priestley et Mill, dans un contexte où l'analyse de la causalité psychologique prend une coloration de plus en plus associationiste.

La «biologisation » opérée par Diderot est aussi à situer dans le contexte du déplacement qu'il opère au sein du spinozisme, puisqu'après tout le corps spinozien n'est pas biologique: pour faire un parallèle rapide, dans l'article «SPINOSISTES » de l'Encyclopédie, quand il distingue entre les «spinosistes anciens» et les «spinosistes modernes », le point crucial est que les seconds sont caractérisés par leur engagement envers une nouvelle théorie de la matière fondée sur l'épigenèse du vivant; soulignons l'idée qu'un spinoziste moderne «suit l'ancien spinozisme dans toutes ses conséquences » mais y ajoute la doctrine biologique de l'épigenèse (Enc. XV, p. 474a). Cette distinction est curieusement proche de celle que propose La Mettrie dans son Abrégé des systèmes,

Plus généralement nous renvoyons au travail très intéressant de Marij van Strien sur les origines du déterminisme laplacien : « On the origins and foundations of Laplacian determinism » (ms., 2013).

${ }^{9}$ Au livre II, chap. 21 : il s'agit en fait de sa critique du libre-arbitre, qu'il revoit dans chacune des cinq éditions de l'Essai, de 1690 à 1701, jusqu'à en faire le chapitre le plus long du livre.

10 Voltaire, Le philosophe ignorant, § xiii, in Euvres complètes, éd. Moland, Paris, 1879, XXVI, p. 55. Pour plus de détails on consultera la thèse de C. Paillard, La Justification de la nécessité : recherche sur le sens et sur les origines du fatalisme moderne, Département de philosophie, Université de Lyon III, 2000. 
entre Épicuriens anciens et Épicuriens modernes (les seconds étant représentés par Gassendi et le médecin Guillaume Lamy, comme nous l'avons analysé ailleurs, au sujet de l'épicurisme médical de La Mettrie'11) : dans les deux cas il s'agit du déplacement d'une ontologie physicaliste vers une biologie, ou une métaphysique biologiquement fondée ${ }^{12}$.

Ce déterminisme qui ne nie pas la spécificité de l'esprit (des actes mentaux), et qui ne se définit pas par opposition au hasard, a quelque chose de souterrain ou d'hétérodoxe dans l'histoire de la philosophie, comme le «matérialisme aléatoire » théorisé par le dernier Althusser. Car cet «autre» déterminisme, pour lequel un coup de dés n'abolit jamais ni le hasard, ni l'esprit et l'action, montre que le stéréotype véhiculé indifféremment par Kant, Husserl ou souvent, la philosophie de l'action contemporaine (notamment les théoriciens de la causalité agentielle) d'un déterminisme aveugle, mécaniste, physicaliste, chez Hobbes, Spinoza ou Laplace n'est après tout que la reprise d'arguments sophistiqués, explicitement chrétiens, formulés notamment par Ralph Cudworth et Samuel Clarke. Ainsi Cudworth oppose la «vie de l'âme» aux «observations extérieures que font les sensations corporelles», prônées par «les épicuriens, les hobbesiens et les athées », qui réduisent la pensée à « un ensemble de mouvements locaux dans le cerveau $»^{13}$. Rejoignant une tradition chrétienne qui aboutit à Schelling et au-delà, la volonté est la vie elle-même - il doit y avoir, écrit Cudworth, « une source ou fontaine vivante de la pensée dans l'âme elle-même » (ibid.). Le premier moteur de l'âme «ne peut être rien d'autre qu'un désir incessant, infatigable et ininterrompu, c'est-à-dire l'amour du bien en tant que tel et la recherche du bonheur » (p. 173). C'est dans le même sens, opposant la pureté de l'agir et du vouloir à l'univers naturel, causal, matériel, que l'Abbé Nicolas-Sylvestre Bergier dira dans son Examen du matérialisme ou Réfutation du système de la nature de 1771, que «Vouloir, c'est agir : si le vouloir n'est

\footnotetext{
11 C.T. Wolfe, « La réduction médicale de la morale chez La Mettrie », in S. Audidière, J.-C. Bourdin, F. Markovits et al., dir., Matérialistes français du XVIIIe siècle. La Mettrie, Helvétius, d'Holbach, Paris, PUF, 2006, p. 45-60.

12 Nous examinons cet aspect de la pensée de Diderot dans «Une biologie clandestine ? Le projet d'un spinozisme biologique chez Diderot», La lettre clandestine $\mathrm{n}^{\circ} 19$ (2011), p. 183-199. Sur les allers et retours entre Épicure, Lucrèce, Spinoza et leurs surinterprétations respectives dans les Lumières radicales, cf. J. Roger, Les sciences de la vie, $3^{\mathrm{e}}$ édition, Paris, Albin Michel, 1993, p. $462-463$ et Y. Citton, L'Envers de la Liberté. L'invention d'un imaginaire spinoziste dans la France des Lumières, Paris, Éditions Amsterdam, 2006, p. 505n.

${ }^{13}$ Cudworth, Treatise on Free-Will (manuscript inédit publié seulement au $19^{\mathrm{e}}$ siècle), $\S 8$, in Cudworth, A Treatise concerning Eternal and Immutable Morality \& A Treatise on Free-Will, éd. S. Hutton, Cambridge, CUP, p. 172.
} 
pas une action dans toute l'exactitude du langage philosophique, il faut créer une nouvelle langue pour les matérialistes $»^{14}$.

Contrairement donc à Cudworth, Clarke, ou plus tard Thomas Reid et Bergier, la figure mutante, lucrétienne, d'un « autre déterminisme », dont l'articulation la plus aboutie est Diderot, cherche à faire une place dans sa visée explicative aux phénomènes non pas «libres » mais «aléatoires » que seraient l'esprit et l'action, au sens d'un univers fondamentalement chaotique, ce qui frise l'oxymore. Quel est alors ce rapport plus subtil à l'esprit, ce déterminisme plus nuancé ? Nous l'avons esquissé ailleurs, concernant Locke et son disciple radical et ami, le déiste Anthony Collins (dont le Philosophical Inquiry Concerning Human Liberty, publié en 1717, soit 13 ans après la mort de son ami Locke, est à mes yeux le «chef-d'œuvre du déterminisme»); ainsi ce qui suit sera de l'ordre d'une série de précisions qui doivent servir à mieux contextualiser Diderot, en tout cas nous le souhaitons ${ }^{15}$.

Rapidement, quand Locke critique le libre-arbitre (sous forme humaniste ou arminienne), il n'affirme pas l'existence d'une nécessité physique universelle, mais plutôt la complexité sous-jacente des volitions humaines, qui ne sont alors jamais indifférentes, notamment à cause de l' « uneasiness » (ce que Leibniz traduira par «inquiétude » mais on peut aussi traduire, comme le fit Coste, par «malaise») qui fonctionne comme un aiguillon : «l'inquiétude est le grand motif qui travaille l'esprit et l'incite à l'action - pour faire bref nous nommerons cela la détermination de la volonté ${ }^{16}$. Locke oppose l'inquiétude la plus pressante à l'autre principe censé déterminer la volonté dans nos actions, le summum bonum, le suprême bien. Aucun jugement intellectuel ne peut susciter une action, comme le fait un désir, «en nous rendant inquiets », littéralement (hors quiétude) (ibid., § 35). L'âme-esprit selon Locke est toujours inquiète : elle court perpétuellement après ce qui comblera ce vide. Mais comment fonder une morale sur une simple compétition hédoniste entre divers stimuli ? Locke répond en revoyant sa copie, introduisant dans sa psychologie de la motivation et de l'action, la notion de suspension

${ }^{14}$ Examen du matérialisme ou Réfutation du système de la nature, 2 vols., Paris, Humblot, 1771, tome II, p. 200, cité par Yves Citton, L'Envers de la Liberté, p. 267.

15 Nous avons proposé une reconstruction détaillée de l'argument lockien tel qu'il s'élabore successivement au cours des éditions de l'Essai, dans «Suspension du désir ou suspension du déterminisme? Le compatibilisme de Locke», in Le déterminisme. Entre sciences et philosophie, dir. P. Charbonnat \& F. Pépin, Paris, Editions Matériologiques, 2012, p. 117-141.

16 Essai concernant l'entendement humain, II.xxi.29. Dans une des critiques les plus «porteuses » de l'Essai, en tout cas pour notre contexte, Leibniz constatait l'absence chez Locke de toute dimension mentale inconsciente (Nouveaux essais, II.xx.6) ; mais dans plusieurs textes écrits en marge de l'Essai, y compris sa correspondance avec Jean Le Clerc, Locke se montre très conscient des niveaux « infimes » de l'inquiétude. 
du désir, probablement tirée de sa lecture de Malebranche (alors que son argumentaire hédoniste remonte à sa lecture de Gassendi ou Bernier dans les années 1676 à Montpellier) ${ }^{17}$. Tiraillés par une multitude de désirs différents, d'inquiétudes, nous avons cependant la capacité cognitive de suspendre leur exécution, de comparer différents «biens » et de prendre une décision.

Bref, Locke élabore une vision de l'esprit qui reconnait sa complexité propre tout en niant un libre-arbitre, mais reste un cocktail instable de volontarisme et d'hédonisme (c'est sa réaction au vers d'Ovide «Video meliora, proboque, deteriora sequor » ${ }^{18}$ ), puisqu'il introduit la suspension du désir. Cette vision sera radicalisée et simplifiée, avec une focalisation toujours plus fine sur l'activité mentale, par Collins, qui accusera précisément Locke d'inconstance en voulant conserver cet élément « suspensif » au sein d'un schéma censé réfuter les notions populaires de la liberté.

Collins, dont l'argumentaire déterministe emprunte librement à Cicéron et Leibniz, ainsi qu'à Hobbes et Spinoza (sans les citer) ${ }^{19}$, fut néanmoins un proche de Locke, durant les dernières années de la vie de ce dernier. En effet, Locke lui écrit que «personne ne comprend mon livre [sc. l'Essai] comme vous », et exprime le souhait d'avoir eu un compagnon comme Collins ${ }^{20}$, ce qui peut aussi signifier un compagnon de route politique, puisque le déisme radical de Collins est clairement un prolongement du projet lockien d'élaborer un «christianisme raisonnable ». Cet éros intellectuel et politique révèle peutêtre un éros d'un autre ordre, car Locke et Collins se donnent aussi la parole sous le rôle

\footnotetext{
17 Mais même chez Bernier on trouve l'idée de suspension : «lorsque nous sommes sur le poinct et en estat d'agir, il est toujours en notre pouvoir de suspendre l'action et de nous arrrester à considérer meurement les choses, en sorte que, distinguant les véritables biens des biens apparens, nous fassions changer les fausses connoissances ou opinions qui pourroient estre dans l'entendement» (Abrégé de la philosophie de M. Gassendi, Lyon, $2^{\mathrm{e}}$ édition 1684 ; éd. S. Murr, Paris, Fayard, 1992, VII, livre III, ch. I, p. 624).

18 Ovide, Métamorphoses, VII, 20-21 (Médée parlant de tuer ses enfants), cit. Locke, Essay, II.xxi.35 (cité également par Descartes, Hobbes, Spinoza - deux fois dans l'Éthique - et Mandeville). Cf. V. Chappell, «Locke on the Freedom of the Will (1992), repris dans Chappell, dir., Locke, Oxford Readings in Philosophy, Oxford, OUP, 1998, p. 86-105.

${ }^{19}$ Collins ne cite pas Spinoza dans le texte qui nous occupe ici (Philosophical Inquiry Concerning Human Liberty, Londres, Robinson, 1717, nombreuses réimpressions), mais dans son débat avec Clarke (Collins, Answer to Clarke's Third Defence, dans Collins \& Clarke, A Letter to Mr. Dodwell [...] together with a defense of an argument made use of in the above letter to Mr. Dodwell, to prove the immateriality and natural immortality of the soul..., Londres, J. Knapton, 1731, p. 386, 387, suscité par la défense (par Collins) du théologien anglican Henry Dodwell, dont les positions sur l'âme et la matière avaient été critiquées par Clarke. Voir Pascal Taranto, Du déisme à l'athéisme: la libre-pensée d'Anthony Collins, Paris, Honoré Champion, 2000, p. 394-399 («La controverse des Lettres à Dodwell: l'âme matérielle »). Collins possédait les Opera posthuma de Spinoza, ainsi que La vie et l'esprit de Spinoza de Lucas, et de nombreux textes clandestins dont Vanini, Bruno, Campanella, Cardan et un ms. inédit de Orobio de Castro.
}

${ }^{20}$ Locke à Collins, lettre 3504 (3.04.1704), in Locke, Correspondence, éd. E.S. De Beer, Oxford, Clarendon Press, 1976-1989, vol. VIII, p. 263. 
de «demoiselles cherchant un beau parti », Locke se disant trop vieux, Collins l'assurant qu'il est jeune et aussi beau qu'une jeune femme, et Locke concluant dans une lettre qu'il ne fit livrer à Collins qu' après sa mort, « vivant, je vous aimais ... »21.

Mais là où Locke défendait encore une vision de l'esprit « à étages », dans laquelle une détermination hédoniste s'accompagnait de la possibilité d'un état de suspension permettant un rapport intellectualiste (et volontariste) à l'action, Collins refuse ce cryptodualisme et immanentise l'espace de l'action et de l'esprit jusqu'au bout : «la notion commune de la liberté est fausse » (Inquiry, p. 22), «si une cause n'est pas une cause nécessaire elle n'est pas une cause du tout » (p. 58), car toute action a un commencement, et tout ce qui possède un commencement possède une cause, ainsi toutes les actions sont causées nécessairement (p. 59).

En fait, suggère Collins, nous sommes toujours en train de vouloir, y compris au niveau infinitésimal (même l'âne de Buridan ne serait jamais indifférent). Collins prend l'exemple d'une promenade : si on me propose d'aller en promenade, ma volonté réagit de suite ; soit affirmativement (je veux aller me promener), soit négativement (je ne le veux pas), ou encore en différant le moment de décision (p. 38). Et malgré l'amour qu'il ressentait pour Locke Collins est très dur face à la vision lockienne d'un état suspensif qui nous hisserait, à la Baron Münchhausen, au-dessus sinon au-delà d'un univers d'enchaînements affectifs, vers un état réflexif et évaluatif. Selon lui, ce moment de suspension n'existe pas : repousser à plus tard une décision, la différer, c'est «avoir la volonté de différer son vouloir sur l'objet proposé »(p. 38). Il affirme clairement que « suspendre son vouloir, c'est encore un acte de la volonté » (p. 39), position qui fut aussi celle de Leibniz dans les Nouveaux essais (non publiés à cette date).

Collins simplifie, radicalise et surtout immanentise le déterminisme un peu timide de Locke; mais au lieu de réduire les fictions de l'esprit à des quantités mesurables au sein d'un univers physicomathématique, il thématise un déterminisme psychologiquement sophistiqué, «volitionnel », qui se soucie plus des volitions et autres actes mentaux que de l'enchaînement global, cosmique, des causes et des effets, par opposition à un déterminisme physicaliste (Hobbes, d'Holbach, Laplace) qui nie l'existence de ce niveau de l'action, ou tend à le réduire à un niveau inférieur.

21 Locke à Collins, lettre 3498, 21/24.03.1704, Correspondence, VIII, p. 253, 255 ; Collins à Locke, lettre 3500, 30.03.1704, 258 ; Locke à Collins, lettre 3648, 23.08.1704 (mais livrée à Collins après la mort de Locke, le 28.10), p. 417. 
D'ailleurs, ce déterminisme non-laplacien, plus psychologiquement nuancé chez Locke et Collins, plus «biologisé » chez Diderot, qui reconnait l'unité causale propre à chaque « organisation » individuelle, peut même reconnaître un acte comme libre, au sens où les motifs ou les impulsions de l'agent forment un lien dans sa chaîne causale, même si ces motifs et ces impulsions sont totalement déterminés (selon la formule de Quine ... qui attribue cette position à Spinoza et Hume ${ }^{22}$ ). Collins, pour sa part, parle de «nécessité morale ", empruntant, dans une stratégie de détournement ou d'appropriation assez audacieuse, l'expression de son principal adversaire, le théologien newtonien Samuel Clarke.

Là où Clarke insistait que l'homme est libre car il est déterminé par une nécessité purement morale, Collins retourne le gant : «Je ne défends que ce que l'on nomme la nécessité morale, au sens où l'homme, qui est un être intelligent et sensible, est déterminé par sa raison et ses sens; et je nie que l'homme soit soumis à une nécessité telle que celle des horloges, des montres et d'autres êtres dépourvus de sensation et d'intelligence, une nécessité absolue, physique ou mécanique » (Inquiry, p. iii). Il ajoute plus loin que même dans un état apparemment indifférent, il existe une détermination proprement affective : « toutes les modifications de l'homme, ses préjugés, ses opinions, son tempérament, ses habitudes doivent être considérées comme des causes au même titre que les objets extérieurs vers lesquels nous porte notre choix» (p. 47). Chez Clarke, au contraire (comme chez Leibniz), la nécessité morale signifie être déterminé par le bien, ce qui n'est donc pas vraiment une nécessité du tout: la nécessité morale, écrit Clarke, «s'accorde évidemment avec la liberté naturelle la plus parfaite $»^{23}$.

Collins formule ici un autre déterminisme puisqu'il cherche à faire une place (avec un brin de mauvaise foi ?) à une nécessité spécifiquement morale. Une définition analogue d'une détermination spécifiquement «morale» se trouve aussi dans l'article «Determination» de la Cyclopaedia de Chambers : «Determinations, again, are either moral or physical : a moral determination is that proceeding from a cause which operates morally $»^{24}$. Pour Collins, nous sommes déterminés par des causes spécifiques, par

22 Quine, «Things and their place in theories », in P.K. Moser \& J.D. Trout, dir., Contemporary Materialism, Londres, Routledge, 1995, p. 199.

${ }^{23}$ Clarke, A Demonstration of the Being and Attributes of God ( $\left(1^{\text {st }}\right.$ Boyle Lecture, 1705), $\S \mathrm{X}$, in Clarke, A Demonstration ... and Other Writings, éd. E. Vailati, Cambridge, Cambridge University Press, 1998, p. 73 ; pour la réponse de Collins, Inquiry, p. 112.

${ }^{24}$ E. Chambers, Cyclopaedia, or a universal dictionary of arts and sciences..., 2 vols., Londres, J. Knapton, 1728 , s.v. «Determination». 
exemple affectives, contrairement aux horloges dont la nécessitation est purement mécanique. On touche là à une aporie ou en tout cas un défi conceptuel de l'ontologie des Lumières matérialistes : quand un autre 'para-spinoziste' comme La Mettrie explique que «demander si la Matière peut penser [...] c'est demander si la Matière peut marquer les heures $\gg^{25}$, est-ce une analogie entre le fonctionnement sensitif d'un homme et son cerveau, et le fonctionnement purement mécanique d'une horloge ? Ou une réduction des propriétés vitales à des propriétés mécaniques ? L'interprétation la plus convaincante est celle de Timo Kaitaro ${ }^{26}$, selon lequel il s'agit d'une analogie fonctionnelle (la pensée ou la sensation est une propriété fonctionnelle d'êtres possédant un certain degré d'organisation, comme la propriété de marquer les heures est une propriété des montres); Collins ne semble pas dire autre chose - même si il ne s'intéresse pas du tout, lui, aux vertus du hasard, se réclamant au contraire du stoïcisme.

Mais il nous reste encore un effort à faire dans notre reconstruction d'un « autre déterminisme », minoritaire, souterrain, partiellement clandestin. Si avec Locke et Collins nous avons vu que ce déterminisme cherchait, non pas à nier l'existence de l'esprit et des actes mentaux, mais à les intégrer dans un monde unifié, causalement clos, il nous faut maintenant passer au dernier point, avec cet épicuro-lucrétien moderne qu'est Diderot ${ }^{27}$. Nous avons signalé l'existence d'une distinction parallèle entre «épicuriens anciens et modernes » (La Mettrie) et « spinozistes anciens et modernes » (Diderot), dans laquelle la différence entre les premiers et les seconds tient chaque fois à une sorte de biologisation. Contrairement à un épicurien ou un spinoziste ancien, un épicurien ou un spinoziste moderne raisonne en termes d'atomes vivants (ou de «corps animés»), ou encore d'épigenèse. Le déterminisme se «physiologise », se «médicalise », se «biologise »: comme le dit La Mettrie, mon désir est comme l'oiseau sur sa branche, toujours prêt à s'envoler, déterminé par des causes spécifiquement organiques $^{28}$. Ces causes organiques, Diderot les concevra comme un prolongement plus matérialiste (ou plus «incarnée »...) de l'enchaînement purement psychologique et affectif étudié par Locke et Collins (avec la nécessité morale). Plusieurs auteurs contemporains de Diderot chercheront à fournir une

${ }^{25}$ La Mettrie, L'Homme-Machine [1748], éd. \& trad. A. Vartanian, Princeton, Princeton University Press, 1960, p. 150.

${ }^{26}$ Kaitaro, «'Man is an admirable machine'-a dangerous idea », in Mécanisme et vitalisme (Lettre de la Maison française d'Oxford, $\mathrm{n}^{\circ}$ spécial 14, 2001), p. 117.

27 Voir la belle étude d'A. Gigandet, «Lucrèce vu en songe. Diderot, Le rêve de D'Alembert et le De rerum natura », Rev. Mét. Mor. vol. 35, n 3 (2002), p. 415-427.

${ }^{28}$ La Mettrie, Discours sur le bonheur, in Cuvres philosophiques, éd. Markovits, Paris, Fayard-Corpus, 1987, I, p. 261. 
version plus concrète du sensualisme, parfois en la présentant comme le soubassement physiologique ou physique (comme chez Hartley) du modèle lockien. De même pour l'accentuation plus forte sur la dimension déterministe: Jean-Baptiste Robinet, par exemple, suggère que les impressions que me transmettent les sens déterminent ma faculté de sentir, qui détermine le contenu de ma pensée, qui détermine ma volonté29.

Si Collins distinguait, mais en suggérant de manière subreptice l'analogie fonctionnelle entre la nécessité morale qui gouverne les mammifères supérieurs et la nécessité purement mécanique et physique qui gouverne les montres, Diderot affirme fortement «Quelle différence d'une montre sensible et vivante à une montre d'or, de fer, d'argent, ou de cuivre ! ${ }^{30}$ (que l'on songe à Collins et sa nécessité morale qui n'est pas celle des horloges). Il refuse de considérer la matière autrement que sentante et pensante ; ainsi quand il reprend le déterminisme volitionnel il le «cérébralise » («pour expliquer le mécanisme de la mémoire il faut regarder la substance molle du cerveau comme une masse d'une cire sensible et vivante ... »: Éléments, p. 470). Comme Collins il refuse toute distinction catégorielle entre la sensation et la raison, mais de manière moins sceptique, plus incarnée que Collins, Diderot affirme qu' «il n’y a qu'une seule opération dans l'homme, c'est sentir. Cette opération [...] n'est jamais libre $»^{31}$, ou qu' « il n'y a pas de bonheur ou de malheur rationnel, mais il y peut y avoir un bonheur ou un malheur animal» (art. DROIT NATUREL, Enc. V, p. 115b). Locke discrètement, Collins définitivement, et de manière plus substantielle Hume et Diderot cherchent à éliminer la coupure homme / animal. Mais remarquons surtout que pour Diderot, cette «biologisation» du bonheur et du malheur, qui est en quelque sorte le renforcement de l'hédonisme de Locke et Collins, n'est pas éliminativiste : il ne nie pas leur existence mais cherche à lui donner un statut légitime (c'est-à-dire compatible avec les données de la science naturelle de son temps).

Comme l'a si bien montré Jacques Chouillet, là où Diderot avait initialement soutenu, avec d'Holbach (et Hobbes, et Laplace), qu' «il n'y a qu'une sorte de causes $[\ldots]$ : les causes physiques », ou que «le monde physique et le monde moral » sont « une

\footnotetext{
29 J.-B. Robinet, De La Nature, Amsterdam, 1761, p. 441-442.

30 Éléments de physiologie, in Diderot, Euvres complètes, dir. Dieckmann-Proust-Varloot, Paris, Hermann, 1975- (dorénavant DPV), vol. XVII, p. 335.

31 Diderot, Considérations sur Hemsterhuis, in Hemsterhuis, Lettre sur l'homme et ses rapports, éd. G. May, Paris, PUF, 1964, p. 155 ; cf. Élém. Physiol. : « la perception naît de la sensation. De la perception la réflexion [...], le jugement. Il n'y a rien de libre dans les opérations intellectuelles [...]» (DPV XVII, p. 335).
} 
seule et même machine ${ }^{32}$, il finit dans la Réfutation d'Helvétius par réclamer une causalité spécifique: comme «je suis homme», «il me faut des causes propres à l'homme ${ }^{33}$. Qu'est-ce qu'un homme pour Diderot ? Certes, ni une essence particulière, ni un être rationnel et libre de type sartrien, mais simplement « une cause une ${ }^{34}$ au sein de la multitude des causes existantes. Or, et là nous retrouvons l'indécidabilité de l'univers lucrétien, au sein de ce « Grand Tout», dans lequel «tout change, tout passe ... il n'y a que le tout qui reste », puisque tout dans la nature est lié et « une volonté sans cause est une chimère »(Diderot, Rêve, DPV XVII, p. 128, et Éléments de physiologie, DPV XVII, p. 483), tout peut changer! L'homme n'est qu'un «effet commun », le monstre un «effet rare»(Rêve, p. 138)! L'espèce elle-même peut disparaitre. Les molécules animées et «folles » s'unissent et se séparent, c'est pour cela que les formes ne sont que des «masques» (Diderot, Éléments, p. 486, 296). Dans cet univers «vaste tripot », nous jouons aux dés, nous sommes des coups de dés de la matière (ibid., p. 516). Goethe, l'ami de la forme, la structure et la totalité, n'avait finalement pas tort de se plaindre : «Étonnant et excellent Diderot, pourquoi toujours utiliser tes grandes forces intellectuelles dans le but de produire du désordre plutôt que de l'ordre ?»35. Déterminisme et hasard se rejoignent, comme dans cette affirmation de Jean La Placette en 1709 : «je suis persuadé que ce qu’Épicure appelait hasard et ce que Spinoza appelle nécessité est la même chose $»^{36}$.

Mais il faut bien voir que ce «désordre » est celui de l'univers vivant dans son imprévisibilité, monstrueuse ou transformiste. Ce que nous avons nommé la «biologisation » du principe de causalité chez Diderot, son non-éliminativisme, explique peut-être pourquoi, en lucrétien moderne, il croit à l'irréductibilité du hasard plutôt que d'affirmer comme d'Holbach que «le hasard est un mot vide de sens ${ }^{37}$. Cela

\footnotetext{
32 Respectivement, lettre à Landois, 29 juin 1756 (DPV IX, 258) et Dieu et l'homme (compte-rendu inédit pour la Corr. littéraire de Dieu et l'homme par P.L. Sissous de Valmire, 1771), DPV XX, 655.

33 Diderot, Réfutation d'Helvétius, in Euvres, vol. 1: Philosophie, éd. L. Versini, Paris, Laffont, coll. Bouquins, 1994, p. 796.

${ }^{34}$ Diderot, Considérations sur Hemsterhuis, p. 157. Cf. J. Chouillet, «Des causes propres à l'homme », in Approches des Lumières. Mélanges J. Fabre, Paris, Klincksieck, 1974, p. 53-62.

35 Goethe, notes de 1799 sur l'Essai sur la peinture de Diderot (Diderots Versuch über die Mahlerei), in Goethe, Écrits sur l'art, trad. J.-M. Schaeffer, Paris, GF-Flammarion, 1996, p. 196.

36 Jean La Placette, Eclaircissement sur quelques difficultés qui naissent de la consideration de la liberté nécessaire pour agir moralement avec une addition où l'on prouve contre Spinosa que nous sommes libres, Amsterdam, 1709, p. 317, cit. in L. Bove, La stratégie du conatus. Affirmation et résistance chez Spinoza, Paris, Vrin, 1996, p. 148, n. 3. Cf. aussi Citton, L'Envers de la liberté, p. 117.

${ }^{37}$ D'Holbach, Système de la nature, II, ch. v, p. 158.
} 
s'accorderait en tout cas avec la fascination qu'éprouve cette pensée pour les monstres (et la génération spontanée), du De rerum natura au Rêve de D'Alembert.

\section{Conclusion}

Le déterminisme a existé sous une forme distincte et significative au sein des Lumières radicales «épicuro-lucréto-spinoziennes ». Rappelons les trois lieux communs au sujet du déterminisme au $18^{\mathrm{e}}$ siècle : (i) il n'existe pas, ou, ce qui revient au même, le mot n'existe pas (on préfèrera alors parler de fatalisme ou de nécessitarisme); (ii) il n'est qu'un pur physicalisme mécaniste ; (iii) il ne propose aucune explication soutenue des phénomènes mentaux et leur intentionnalité.

Nous avons voulu montrer que ces trois lieux communs sont faux: le déterminisme existe, sous plusieurs formes - cérébrales, psychologiques, organismiques qui toutes ne correspondent pas à la définition usuelle et canonique d'un système de points mathématico-physiques dont on pourra calculer toutes les positions et permutations possibles, et qui toutes, a fortiori chez Locke et Collins, reconnaissent l'existence de l'action et des phénomènes intentionnels s'y rapportant. Cet « autre déterminisme », ce monstre du déterminisme, est d'une part spécifiquement psychologique ou « volitionnel» (Locke, Collins) ; d'autre part, avec les atomes vivants, l'épigenèse, les monstres et la détermination de l'organisation par des causes spécifiques (comme un prolongement organismique chez Diderot de la nécessité morale conceptualisée par Collins), c'est un déterminisme biologisant, qui reconnait les particularités des chaînes d'action et réaction corporelles et psychologiques. Qu'on le qualifie de lucrétien, clandestin, biologisant, ou post-lockien, il y a donc dans cette tendance qui culmine chez Diderot quelque chose de radicalement non-physicaliste, qui ne remplace pas la causalité mentale ou biologique par une pure causalité physique, mathématisable et donc calculable (par opposition à la forme plus physicaliste de déterminisme, prédominante chez d'Holbach et Laplace par exemple). Parfois - pas chez Locke ou Collins, mais nettement chez Diderot, il demeure quelque chose de 'hasardeux', d'imprévisible, quoique cette imprévisibilité n'est pas le dernier mot de l'auteur de Jacques le fataliste, qui propose dans l'article VOLONTE de l'Encyclopédie, une expérience dans laquelle «Si l'on pouvait supposer cent mille hommes tous absolument conditionnés de même, et qu'on leur présentât un même objet de désir ou 
d'aversion, ils le désireraient tous, et tous de la même manière, ou le rejetteraient tous, et tous de la même manière $»^{38}$. Néanmoins, on est loin du calcul et de la prévisibilité.

Charles T. WOLFE

Department of Philosophy and Moral Sciences

\& Sarton Centre for History of Science

Ghent University

Blandijnberg 2,

B-9000 Gand Belgique

ctwolfe1@gmail.com

38 Diderot, article «Volonte (Gram. \& Philosophie morale) », Enc. XVII, p. 454b ; même exemple mais féminisé («si je suppose cent mille femmes... ») dans l'article MACHINAL, Enc. IX, p. 794a. 